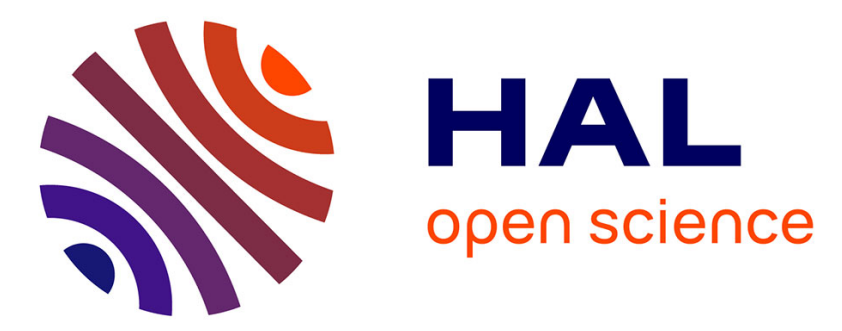

\title{
Principe de commande numérique temps réel d'un onduleur à modulation de largeur d'impulsion sur charges non linéaires
}

\author{
Pascal Maussion, Jean Faucher, Marcel Grandpierre
}

\section{To cite this version:}

Pascal Maussion, Jean Faucher, Marcel Grandpierre. Principe de commande numérique temps réel d'un onduleur à modulation de largeur d'impulsion sur charges non linéaires. Journal de Physique III, 1994, 4 (6), pp.1101-1119. 10.1051/jp3:1994189 . jpa-00249169

\section{HAL Id: jpa-00249169 https://hal.science/jpa-00249169}

Submitted on 1 Jan 1994

HAL is a multi-disciplinary open access archive for the deposit and dissemination of scientific research documents, whether they are published or not. The documents may come from teaching and research institutions in France or abroad, or from public or private research centers.
L'archive ouverte pluridisciplinaire HAL, est destinée au dépôt et à la diffusion de documents scientifiques de niveau recherche, publiés ou non, émanant des établissements d'enseignement et de recherche français ou étrangers, des laboratoires publics ou privés. 


\title{
Principe de commande numérique temps réel d'un onduleur à modulation de largeur d'impulsion sur charges non linéaires
}

\author{
Pascal Maussion, Jean Faucher et Marcel Grandpierre \\ Institut National Polytechnique de Toulouse. Ecole Nationale Supérieure d'Electrotechnıque, \\ d'Electronique, d'Informatique et d'Hydraulique de Toulouse, Laboratoire d'Electrotechnique et \\ d'Electronique Industrielle, Unité Associée au C.N.R.S. n 847, 2 rue Charles Camichel, 31071 \\ Toulouse Cedex, France
}

(Reçu le 21 juillet 1992, révisé le 13 janvier 1994, accepté le 17 mars 1994)

\begin{abstract}
Résumé. - Cet article propose une méthode nouvelle et originale pour la commande numérique temps réel d'un onduleur MLI sur charges non linéaires. Un asservissement de la valeur moyenne instantanée de la tension de sortie est réalisé par l'intermédiaire d'un correcteur proportionnel dérivé et d'une boucle d'anticipation. Les variations de la charge sont prises en compte par précompensation ce qui permet d'obtenir de bons résultats vérifiés par simulation, tant pour le taux de distorsion harmonique que pour la valeur du terme fondamental.
\end{abstract}

Abstract. - This paper proposes a new method for the real tıme digital control of a P.W.M. inverter, used in U.P.S. (Uninterruptible Power Supply). The output voltage is compared to a sinusoidal reference, to compute in real time through a digital controller, the pulse width of the same interval. This strategy is verified through computer simulations and provides a very fast compensation of disturbances, caused by non linear loads such as rectifiers and triac loads, with low T.H.D. (Total Harmonic Distortion) and fondamental control.

\section{Nomenclature.}

E tension d'alimentation de l'ondulateur.

$V_{\mathrm{AB}} \quad$ tension de sortie de l'onduleur après filtrage.

$V_{1} \quad$ valeur fondamentale de la tension $V_{\mathrm{AB}}$.

$V_{\mathrm{M}} \quad$ valeur moyenne de la tension $V_{\mathrm{AB}}$.

$T_{\mathrm{e}} \quad$ période d'échantillonnage.

$n \quad$ numéro de la période du signal de sortie $V_{\mathrm{AB}}(t)$ étudiée.

$k \quad$ numéro de l'intervalle de modulation et d'échantillonnage.

$T_{k} \quad$ largeur de l'impulsion de commande dans l'intervalle de modulation $k$.

$z \quad$ variable de la transformée en $z$.

$p \quad$ variable de la transformée de Laplace. 
$t_{c}$ temps séparant deux mesures successives de la tension $V_{\mathrm{AB}}$.

$\varepsilon_{k n} \quad$ grandeur d'erreur à l'instant $k$ dans la période $n$.

$U_{k} \quad$ grandeur de commande à l'instant $k$.

$d_{0} \quad 5 \quad$ coefficients de l'algorithme de contrôle.

\section{Introduction.}

L'emploi de convertisseurs statiques de plus en plus puissants pour la réalisation de réseaux alternatifs au moyen d'onduleurs de tension à Modulation de Largeur d'Impulsion (MLI) a dónné une importance croissante au problème de l'optimisation de leur commande. Les techniques numériques permettent désormais d'intégrer de nombreuses contraintes, liées aux critères de réglage ou à l'environnement direct des onduleurs (alimentation, imperfections technologiques, charge, etc.).

Le partage d'un même réseau par plusieurs utilisateurs peut poser des problèmes de pollution électrique. En effet, chaque équipement influence la source d'alimentation à laquelle il est relié, agissant en conséquence sur les autres équipements connectés en parallèle, cas des charges non linéaires. Le développement de ce type de charge est lié au fonctionnement en commutation des interrupteurs statiques qui crée des courants harmoniques engendrant des distorsions de l'onde de sortie et modifiant ses caractéristiques. Les effets de ces composantes harmoniques sont nombreux (échauffements, vibrations, pollution électrique, fragilisation.. ) [1. 2] et suffisamment nuisibles pour que l'on envisage de les réduire. C'est là l'objet de cet article.

Quelle commande pour les onduleurs de tension MLI sur charges non linéaires ? La méthode détaillée dans cette étude apporte quelques nouveaux éléments de réponse à cette question. Elle se situe dans l'évolution des techniques de commande, depuis les premières stratégies en boucle ouverte hors ligne sur charge linéaire, jusqu'aux commandes temps réel en boucle fermée sur charges non linéaires. Ces développements sont possibles grâce aux progrès concomitants des interrupteurs statiques et des microprocesseurs. Les temps de commutation des nouveaux interrupteurs statiques permettent l'augmentation des fréquences de découpage. La rapidité et la précision de calcul des microprocesseurs rendent désormais possible des commandes numériques d'onduleur en temps réel.

Les constantes de temps du processus à contrôler étant plus faibles que celles des systèmes électromécaniques classiques, un impératif de rapidité nous guidera. Pour le satisfaire, nous nous efforcerons de rechercher l'efficacité dans la simplicité.

\section{Sur la commande des onduleurs à MLI : spécificité des charges non linéaires.}

2.1 Commande en Boucle Ouverte. - De très nombreuses méthodes ont été proposées pour optimiser les formes d'onde délivrées en MLI. Universellement reconnus comme la référence dans ce domaine, les premiers travaux [3] reposaient sur un calcul préalable horsligne des angles de commutation. Ce principe permet l'élimination sélective d'harmoniques et une minimisation du taux de distorsion pour un motif de modulation et des conditions de fonctionnement données, mais il impose la résolution d’équations non linéaires.

Chaque motif est ainsi adapté aux conditions de fonctionnement pour lequel il a été calculé. Toute modification des grandeurs de réglage (amplitude, phase du fondamental) ou de l'environnement de l'onduleur (tension d'alimentation, charge...) entraîne une inadéquation du motif de commande fixé. Plusieurs motifs de modulation différents peuvent être mémorisés [4]. mais la capacité de stockage ne sera jamais suffisante pour répondre à l'infinité de cas possibles, surtout pour les variations de la charge. 
Mais toutes ces méthodes désormais classiques, présentent l'inconvénient d'être inadaptées aux charges non linéaires rapidement variables. Il devient alors indispensable d'envisager une commande qui prenne en compte les variations de la charge le plus rapidement et le plus simplement possible. à partir des conséquences observées sur la grandeur de sortie de l'onduleur, forme d'onde, distorsion.

2.2 COMMANDE EN BOUCLE FERMÉE. - Des solutions analogiques ont été proposées pour prendre en compte les spécificités des charges non linéaires [5]. Mais l'amélioration des performances n'est ici encore obtenue que grâce à un système de filtrage de puissance, un second convertisseur est nécessaire afin d'injecter directement sur le réseau alternatif, un courant de compensation.

Si la fonction filtrage peut-être transférée du matériel vers le logiciel, une diminution significative de la taille des composants pourra être envisagée. Cette amélioration permettra de réduire la masse donc le volume et le coût de l'ensemble du système. Ce critère prend une importance particulière pour les alimentations autonomes embarquées.

La réalisation numérique de la commande d'onduleur à modulation de largeur d'impulsion sur charge non linéaire doit prendre en compte les contraintes suivantes:

- La distorsion harmonique en régime permanent et en régime transitoire doit être sinon rigoureusement annulée, tout au moins minimisée ;

- le fondamental de la tension de sortie doit être réglé à une valeur de référence.

L'étude présentée dans cet article fait suite à des travaux qui furent réalisés au L.E.E.I. de Toulouse dans le cadre d'un contrat avec la DRET en 1987 [6]. Ces travaux reposaient sur l'analyse spectrale du signal de sortie et le calcul hors-ligne itératif des angles de commutation. Cette méthode permet une prise en compte globale des déformations, elle est donc particulièrement bien adaptée à une optimisation du régime permanent. Mais le temps de calcul qu'elle nécessite, même compte tenu des performances actuelles des microprocesseurs, la rend inappropriée à l'amélioration des régimes transitoires. La figure 1 présente mis bout à bout, les résultats des phases d'optimisation successives lors d'un fonctionnement sur charge non linéaire.

Les résultats obtenus furent très satisfaisants quant à la qualité de l'onde. Mais cette optimisation n'était traitée qu'en temps différé et diverses stratégies type poursuite de sinusoïde temps réel en boucle fermée ont été proposées pour pallier cet inconvénient.

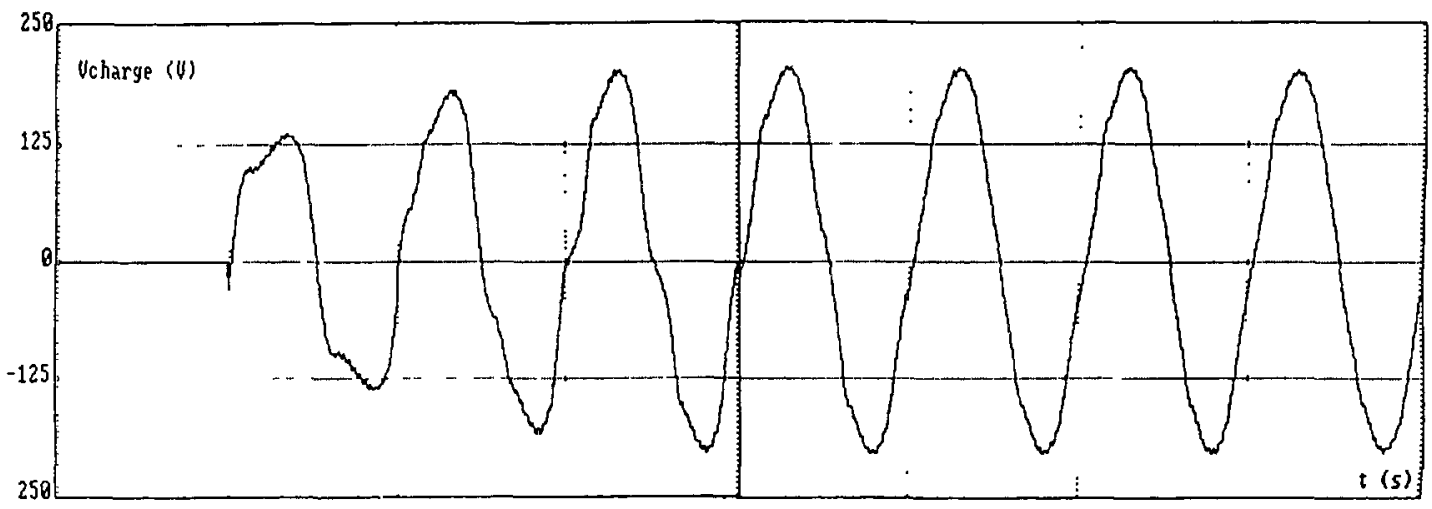

Fig. 1. - Evolution de la tension de sortie lors de l'optimisation globale hors-ligne.

[Effects of an off-line step by step optimization on the output waveform.] 
- Un contrôleur à hystérésis [7] permet la réalisation d'une commande «bang-bang » avec asservissement de la tension de sortie de l'onduleur à une référence sinusoïdale, par adaptation de la largeur de l'hystérésis à la valeur instantanée de la tension de référence. Mais la fréquence des commutations dépend alors de la largeur de l'hystérésis.

- Une commande numérique temps réel a été proposée à partir de la modélisation de la charge par les équations d'état [8] appelée «Dead Beat Control ", donne des résultats satisfaisants sur des charges non linéaires résistives peu contraignantes grâce à des correcteurs calculés hors-ligne à partir de cette modélisation. Elle permet également le contrôle de deux convertisseurs statiques en parallèle sur une même charge [8].

La nouvelle méthode de compensation proposée dans cette étude, se base sur un asservissement de la tension de sortie de l'onduleur par poursuite d'une référence variable dans chaque intervalle de modulation. Nous étudierons le cas d’un signal sinusoïdal, mais la commande proposée pourrait s'adapter à tout type de consigne.

\section{Principes de la commande temps réel par asservissement de la valeur moyenne instantanée.}

3.1 DesCRIPTION GÉNÉRALE DU CADRE DE L'ÉTUDE. - La figure 2 présente le schéma du montage étudié. Un onduleur de tension monophasé alimente une charge non linéaire par l'intermédiaire d'un filtre LC destiné à éliminer les harmoniques dus au découpage. Le comportement de ce montage est décrit par une équation différentielle du $2^{\mathrm{e}}$ ordre dont les coefficients varient en fonction de la charge.

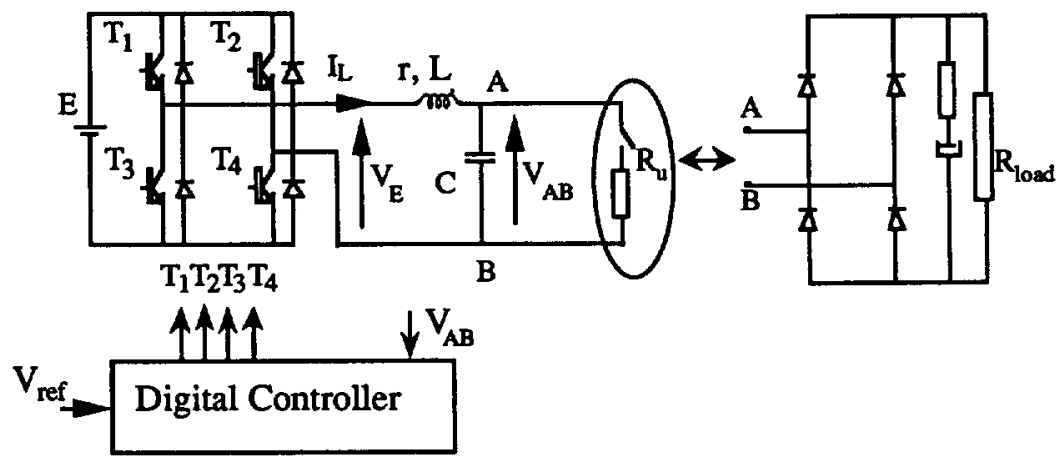

Fig. 2. - Schéma du montage étudié. $E=220 \mathrm{v}, \mathrm{L}=1,76 \mathrm{mH}, \mathrm{r}=0,5 \Omega$. Fréquence de référence : $50 \mathrm{~Hz}, R_{u}=3 \Omega, \mathrm{V}_{\mathrm{rel}}=100 \mathrm{v}, C=280 \mu \mathrm{F}$, Fréquence d'échantillonnage $: f_{\mathrm{c}}=3,2 \mathrm{kHz}$.

L'étude sera conduite au moyen d'un programme de simulation (algorithme RK2), structuré selon la méthodologie SOSIE [10]. Il prend en compte l'aspect structurel de la réalisation, c'est-à-dire les effets de la numérisation dus au convertisseur analogique-numérique et au calcul sur microprocesseur qui seront choisis.

Le redressement-filtrage par pont de diodes et capacité en tête, ou la charge de type triac sont des charges non linéaires, puisque variables plusieurs fois par période du signal de sortie de l'onduleur $V_{\mathrm{AB}}$. Elles sont particulièrement contraignantes du point de vue harmonique, puisque le courant de sortie de l'onduleur est alors fortement impulsionnel durant le temps de conduction des diodes de redressement. 
Le modèle de charge non linéaire retenu pour la simulation est constitué d'une résistance en série avec un interrupteur commandé 4 fois par période. Une étude précédente [6], a montré que cette simplification du modèle rendait compte fidèlement des différents phénomènes. Il sera retenu en raison de sa simplicité de programmation et d'interprétation des formes d'ondes.

3.2 NéCESSitÉ DE LA COMPENSATION. - Les charges non linéaires déforment la tension de sortie de l'onduleur. Un exemple est donné sur la figure 3. Les ondes sont caractérisées par un taux de distorsion important et le fondamental ne peut être maintenu à sa valeur nominale, dans cet exemple $100 \mathrm{~V}$. Ces résultats ont été vérifiés avec le logiciel de simulation S.C.R.I.P.T. [11], afin de s'assurer de la validité de l'algorithme en boucle ouverte.

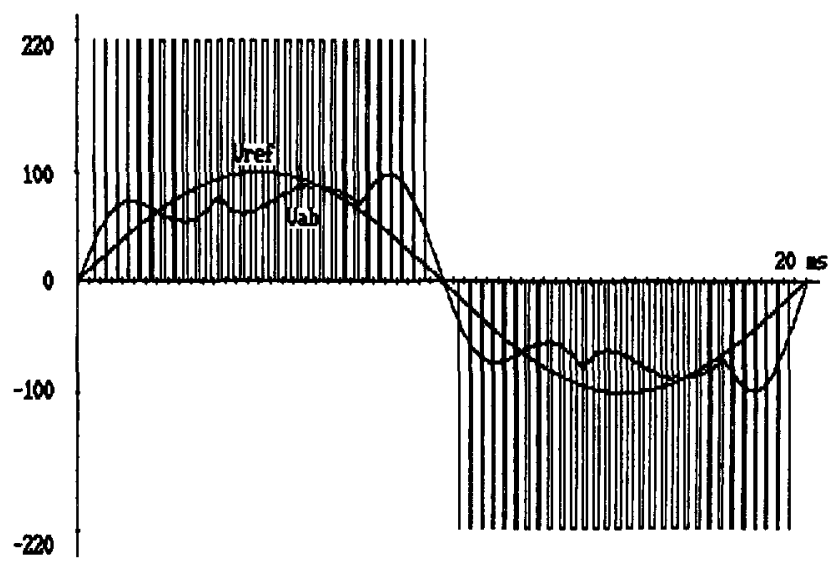

a)

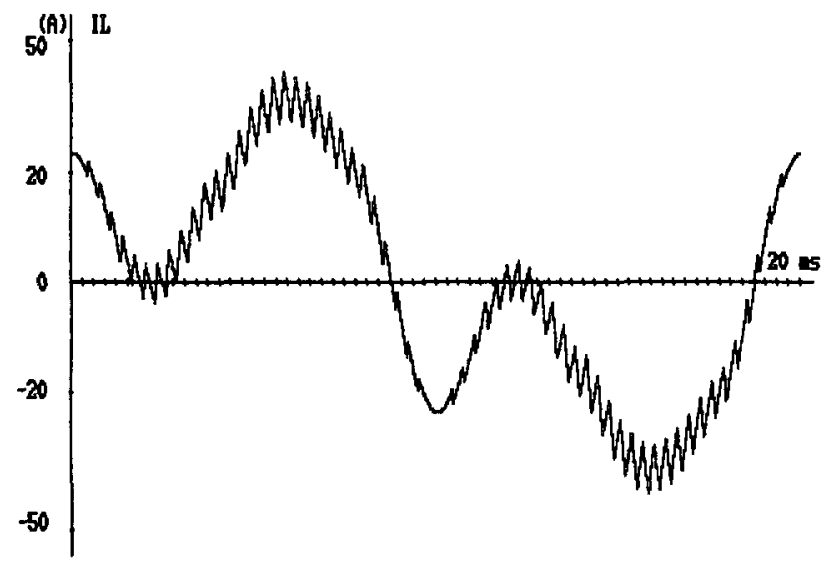

b)

Fig. 3. - a) Tension de sortie du filtre $V_{\mathrm{AB}}$. b) Courant dans l'inductance $I_{\mathrm{L}}$. $3 \Omega$ sur $\left[70^{\circ}-140^{\circ}\right]$ et $\left[250^{\circ}\right.$ $\left.320^{\circ}\right], v_{1}=93,6 \mathrm{~V}, \tau=37,8 \%$.

[a) Output voltage $V_{\mathrm{AB}}$. b) Line current $I_{\mathrm{L}} .3 \Omega$ over $\left[70^{\circ}-140^{\circ}\right]$ and $\left[250^{\circ}-320^{\circ}\right] . V_{1}=93.6 \mathrm{~V}$, $\tau=37.8 \%$.] 
Le taux de distorsion est défini par la relation

$$
\tau=\frac{\sqrt{\sum_{i=2}^{\infty} V_{l}^{2}}}{V_{1}}
$$

Les essais sans régulation, en boucle ouverte, montrent l'effet d'une charge non linéaire sur la forme d'onde de sortie. Nous observons une chute brutale de la tension lors de l'appel de courant, quand la charge est connectée et une oscillation importante, vers $140^{\circ}$ lorsqu'elle est déconnectée alors que le courant n'est pas nul dans l'inductance. le filtre se trouvant ainsi placé dans les conditions de l'amortissement minimal. Il convient donc d'éliminer ces effets importants, dont les conséquences sont nombreuses et parfois coûteuses ou dommageables pour les équipements connectés sur le réseau alternatif $[1,2]$. La solution proposée dans cet article, consiste à réaliser un asservissement de la tension de sortie dans chacun des intervalles de modulation.

3.3 VAlEUR MOYENNE inSTANTANÉE. - Le système devra compenser les effets des charges non linéaires pour que la grandeur de sortie $V_{\mathrm{AB}}(t)$ suive une consigne variable, quelles que soient les perturbations extérieures. Le signal de consigne $V_{\text {ret }}$ pourrait être tout à fait quelconque, mais nous nous limiterons au cas d'une onde sinusoïdale. Un microprocesseur devra donc calculer en temps réel à chaque instant d'échantillonnage, $t=k T_{\mathrm{e}}$, la largeur $T_{h}$ des impulsions de tension produites par l'onduleur.

Dans chaque intervalle de modulation, nous choisissons d'asservir la valeur moyenne de la tension de sortie et non pas la valeur instantanée de celle-ci. Cette valeur moyenne dans chacun des 64 intervalles de modulation est appelée « valeur moyenne instantanée » [12]. L'originalité de ces travaux est due pour une part à ce choix et aux propriétés stabilisatrices, intrinsèques à ce type de retour. Cette valeur moyenne instantanée $V_{\mathrm{M}}$ de la tension de sortie sera estimée par interpolation à partir de la valeur instantanée et de la pente $\Delta V / \Delta T$ à chaque instant d'échantillonnage. En effet, il est possible de linéariser la courbe par morceaux et d'assimiler la valeur instantanée au milieu de l'intervalle à la valeur moyenne sur cet intervalle, si celui-ci est suffisamment petit.

La grandeur de retour $V_{\mathrm{M}}\left(k T_{\mathrm{e}}\right)$ sera en elle-même porteuse d'une information sur la vitesse d'évolution de la tension de sortie de l'onduleur au travers de sa dérivée, comme lors d'un retour tachymétrique dans un asservissement de position. La poursuite de la référence sinusoïdale n'en sera que plus précise en régime dynamique, grâce à cette sorte de retour dérivé qui est en quelque sorte intrinsèque [13] au type de retour utilisé.

L'estimation de la pente entre deux instants d'échantillonnage successifs, permettrait le calcul de la valeur moyenne instantanée. Mais cette estimation pourrait être plus précise en réduisant le $\Delta T$ à une valeur optimale $t_{c}$. Nous réaliserons dès lors ce calcul par suréchantillonnage (Fig. 4). Cette méthode de mesure donne de bons résultats, puisqu'elle permet d'obtenir une valeur moyenne calculée $V_{\mathrm{AB} \text { moy calculée }}$ très proche de la valeur moyenne vraie $V$ AB moy vrale

La formule (2) ci-après détaille le calcul exact de la valeur moyenne estimée, tel qu'il sera implanté sur le calculateur pour la simulation, puis sur microprocesseur.

$$
V_{\mathrm{M}}\left(k T_{\mathrm{e}}\right)=V_{\mathrm{AB}}\left(k T_{\mathrm{e}}\right)+\frac{T_{\mathrm{e}}}{2} \times \frac{V_{\mathrm{AB}}\left(k T_{\mathrm{e}}\right)-V_{\mathrm{AB}}\left(k T_{\mathrm{e}}-t_{\mathrm{c}}\right)}{t_{\mathrm{c}}}
$$

La valeur du temps $t_{\mathrm{c}}$ séparant deux instants d'échantillonnage pour le calcul de la pente sera le 


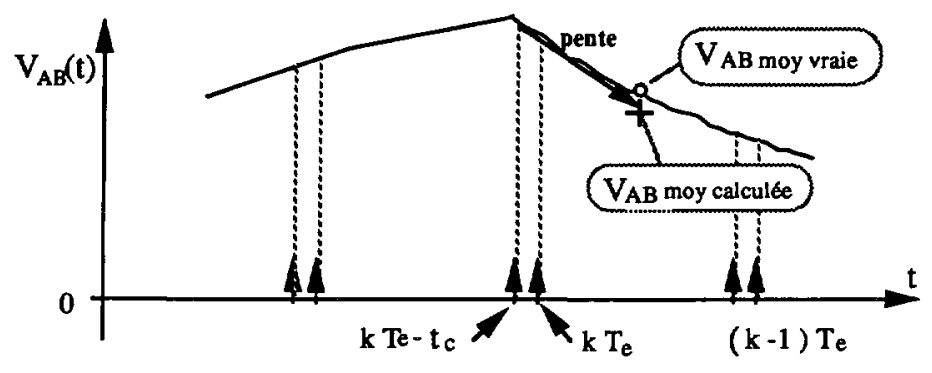

Fig. 4. - Mesure de pente par sur-échantillonnage.

[Slope measurement by over-sampling.]

fruit d'un compromis. Elle doit permettre d'obtenir une poursuite satisfaisante de la référence sinusoïdale, tout en maintenant les erreurs d'arrondis dus à la numérisation et aux calculs, dans un domaine de valeurs acceptables. Nous poserons en ce début d'étude $t_{\mathrm{c}}=30 \mu \mathrm{s}$, c'est-à-dire environ $1 / 10^{\circ}$ de la période d'échantillonnage. Nous reviendrons sur ce choix pour une phase d'optimisation du temps $t_{\mathrm{c}}$ lors du fonctionnement en boucle fermée avec régulation.

3.4 Modélisation de la mesure de la valeur moyenne instantanée. - A partir des considérations précédentes. la modélisation de la boucle d'asservissement n'est ni immédiate ni habituelle de part la nature même de la grandeur asservie et la façon de la "mesurer ».

L'idée directrice pour cette étape de modélisation de la boucle de régulation par la transformation en $z$, consiste à séparer fictivement les deux grandeurs mesurées : le signal de sortie et sa pente. La figure 5 indique le mode opératoire de ce calcul, en isolant d'abord une transmittance pour la grandeur de sortie du processus $V_{\mathrm{AB}}(t), G_{1}(z)=V_{\mathrm{AB}}(z) / U(z)$ puis une transmittance de retour pour la dérivée de la sortie $G_{1}(z)=V_{\mathrm{AB}}^{\prime}(z) / U(z)$, en tenant compte du bloqueur d'ordre 0 . La transmittance $G_{1}(z)$ fait apparaître un terme en $p . H(p)$ qui exprime ici la nature du signal mesuré sur cette voie. Le cas le plus défavorable en ce qui concerne la stabilité du système est représenté par le fonctionnement du filtre LC à vide. C'est donc ce cas que nous allons identifier.

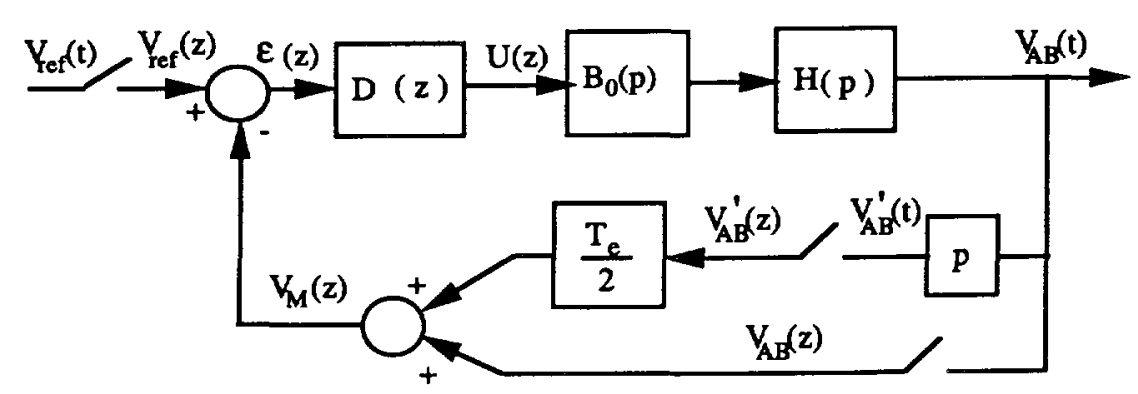

Fig. 5. - Schémas-blocs de l'asservissement.

[Bloc diagram of the feedback control.]

Nous pouvons calculer les transmittances échantillonnées $G_{1}(z)$ et $G_{2}(z)$, puis la fonction de transfert en boucle fermée $H_{\mathrm{BF}}(z)$ pour une période d'échantillonnage de $312,5 \mu \mathrm{s}$, correspondant à 64 intervalles de modulation par période de $20 \mathrm{~ms}$ (fréquence de base de $50 \mathrm{~Hz}$ ). Il 
vieil., compte tenu des valeurs numériques de la figure 1 :

$$
\begin{gathered}
G_{1}(z)=\frac{0,0946 z-0,0918}{z^{2}-1,7285 z+0,915} \\
G_{2}(z)=\frac{586,95 z-586.95}{z^{2}-1,7285 z+0,915}
\end{gathered}
$$

Il est alors possible de modifier et simplifier le schéma-blocs de l'asservissement échantillonné figure 6, en faisant apparaître d'abord la grandeur $V_{\mathrm{AB}}$ comme grandeur de sortie et donc sa valeur moyenne instantanée $V_{\mathrm{M}}$ comme grandeur de retour.

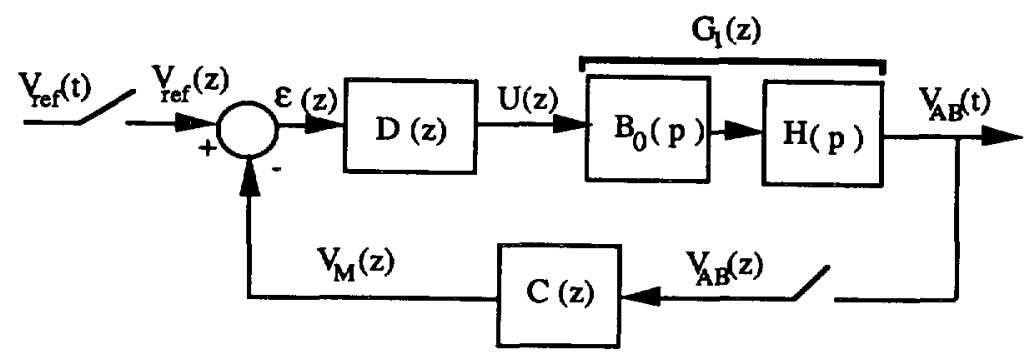

Fig. 6. - Schéma-blocs simplifié de l'asservissement.

[Simplified bloc diagram of the feedback control.]

La grandeur de retour valeur moyenne instantanée $V_{\mathrm{M}}$ sera issue d'une transmittance de filtrage $C(z)$ et élaborée à partir de la tension $V_{\mathrm{AB}}$. Dans ces conditions, il vient :

$$
\begin{aligned}
& V_{\mathrm{M}}(z)=G_{1}(z) \cdot U(z)+\frac{T_{\mathrm{e}}}{2} \cdot G_{2}(z) \cdot U(z) \\
& V_{\mathrm{M}}(z)=G_{1}(z) \cdot U(z) \cdot\left[1+\frac{T_{\mathrm{e}}}{2} \cdot \frac{G_{2}(z)}{G_{1}(z)}\right] \\
& V_{\mathrm{M}}(z)=G_{1}(z) \cdot U(z) \cdot C(z) \operatorname{avec} C(z)=1+\frac{T_{\mathrm{e}}}{2} \cdot \frac{G_{2}(z)}{G_{1}(z)} .
\end{aligned}
$$

3.5 MÉThOdE DU TALON. - Nous allons envisager un type de commande qui offre quelques avantages par rapport à la correction série classique par un correcteur $D(z)$.

Il s`agit du principe de commande par chaîne d'anticipation [14-16] dont le schéma-blocs est représenté sur la figure 7 et qui peut assurer une erreur quasi nulle en régime permanent sans

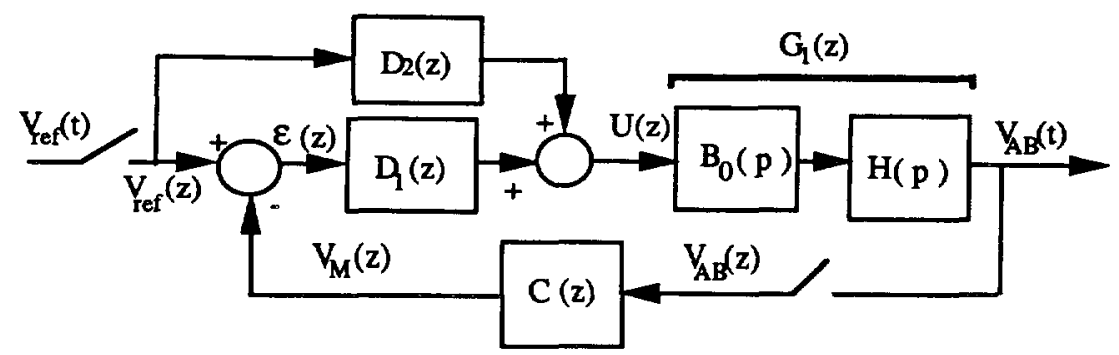

Fig. 7. - Schéma-blocs de l'asservissement par chaîne d'anticipation.

[Block diagram of the feedback control with feedforward loop.] 
terme intégral. Dans le cas présent d'une référence continuement variable, l'action intégrale présente peu d'intérêt, contrairement aux cas plus classiques d'entrée en échelon ou en rampe. Le contrôle de l'ensemble est assuré par l'intermédiaire de deux boucles $D_{1}$ et $D_{2}$ dédiées chacune à une fonction spécifique: l'asservissement pour $D_{2}$ et la régulation pour $D_{1}$. Le correcteur $D_{1}$ stabilisera le système et fixera le comportement en régulation vis-à-vis des perturbations créées par les charges non linéaires. Il travaille sur les variations de l'erreur autour d'un point de repos créé via la transmittance $D_{2}$.

Il ne s'agit pas ici de la poursuite d'un modèle de référence, mais d'une stratégie plus simple visant la rapidité du contrôle dans l'optique d'une réalisation temps réel. De plus, le modèle même du système peut être variable dans le cas de la connexion en parallèle sur la sortie de l'onduleur, de différentes charges, linéaires ou non linéaires : charge R ou R-L, charge type gradateur, redresseur avec filtrage par condensateur. L'adaptation du correcteur $D_{2}(z)$ aux variations du modèle demanderait une stratégie plus complexe qui sort du domaine de cette étude. La technique retenue ici repose sur le principe du talon [16], afin de réaliser l'asservissement de la grandeur de sortie $V_{\mathrm{AB}}\left(k T_{\mathrm{e}}\right)$ à la consigne sinusoïdale $V_{\text {ref }}\left(k T_{\mathrm{e}}\right)$. Ce type de contrôle présente deux avantages par rapport à une régulation ou à un asservissement cascade classiques, à grandeur de commande $U(z)$ fixe :

- pour une même valeur de l'erreur, le gain statique de la transmittance $D_{1}(z)$ sera moins grand dans la structure avec chaîne d'anticipation que dans le cas classique, d'où une meilleure stabilité ;

- pour une même valeur du gain statique de la transmittance $D_{1}(z)$, l'erreur sera plus faible d'où une meilleure précision.

De plus, par rapport à une commande classique, la chaîne d'anticipation possède un degré de liberté supplémentaire. Le correcteur $D_{1}(z)$ permet d'imposer le comportement en régulation par rapport à la perturbation $v(t)$, qui sera ici une variation de la charge. Il est alors encore possible d'imposer la transmittance en boucle fermée en agissant sur le terme $D_{2}(z)$. Les deux degrés de liberté permettent donc deux réglages séparés pour le fonctionnement en régulation et en asservissement. Cette méthode présente l'avantage d'annuler l'erreur en régime permanent sans avoir recours à un terme intégral.

3.6 CAlCul DES CORRECTEURS. - Vu la nature de la charge et son comportement oscillatoire, la correction envisagée sera de type proportionnelle-dérivée. Dans ces conditions la transmittance de compensation $D_{1}(z)$ peut s'écrire :

$$
D_{1}(z)=d_{0}+d_{1} \cdot z^{-1}
$$

La manière la plus simple de réaliser la transmittance de compensation $D_{2}(z)$ est une constante $d_{2}$ qui sera déterminée en fonction d'un critère de précision en boucle fermée. Ce choix simple de la transmittance $D_{2}(z)$ est lié pour une part à l'objectif de rapidité que nous nous sommes fixé au début de cette étude. D'autre part, il serait possible de compenser les pôles de la transmittance du système $G_{1}(z)$, par le choix de zéros appropriés dans la transmittance de la boucle d'anticipation $D_{2}(z)$. Mais nous ne retiendrons pas cette stratégie en raison des fortes variations du modèle. La compensation exacte de la transmittance du système $G_{1}(z)$ n'est donc pas possible. Les résultats de simulation nous prouveront l'efficacité du choix, $d_{2}=$ constante.

L'algorithme de compensation et de commande fournit la valeur de la tension appliquée à l'onduleur à partir de la référence $V_{\text {ref } k}$ à chaque instant d'échantillonnage $k T_{\mathrm{e}}$ et des erreurs sur la valeur moyenne instantanée $\varepsilon_{k}$ et $\varepsilon_{k-1}$.

$$
U_{k}=d_{2} \cdot V_{\text {ref } k}+d_{0} \cdot \varepsilon_{k}+d_{1} \cdot \varepsilon_{k-1} \cdot
$$


Le schéma-blocs figure 7 permet de déterminer la transmittance du système en boucle fermée (8) :

$$
\frac{V_{\mathrm{AB}}(z)}{V_{\text {ret }(z)}}=\frac{\left(D_{1}(z)+D_{2}(z)\right) \cdot G_{1}(z)}{1+G_{1}(z) \cdot C(z)}
$$

Nous utiliserons pour le calcul du correcteur proportionnel-dérivé $\left(d_{0}, d_{1}\right)$, la méthode du placement des pôles et des zéros [17]. Nous imposerons le comportement dynamique du système, par identification à une fonction de transfert, tel que le dépassement soit faible et les réponses, indicielle ou impulsionnelle, les plus rapides possible, compte tenu de la période d'échantillonnage fixée : $\zeta=0,7$ et $\omega_{p} \leqslant \pi / 4 T_{\mathrm{e}}$. Nous effectuons en premier lieu un calcul à partir de la fonction de transfert à vide qui représente le cas le plus défavorable. En développant l'expression (8) en remplaçant $D_{1}(z)$ par sa valeur (6) et en tenant compte de l'expression (7), avec $D_{2}(z)=d_{2}$, il vient finalement (9):

$$
\begin{aligned}
& H_{\mathrm{BF}}= \\
& =\frac{0,0952 \cdot\left(d_{0}+d_{2}\right) \cdot z^{2}+z \cdot\left[\left(d_{0}+d_{2}\right) \cdot 0,09235+0,0952 \cdot d_{1}\right]+0,09235 \cdot d_{1}}{\left[z^{3}+z^{2}\left(0,1874 \cdot d_{0}-1,727\right)+\left(0,1864 \cdot d_{1}+0,915+0,0001 \cdot d_{0}\right) \cdot z-5,9 \times 10^{-4} d_{1}\right]}
\end{aligned}
$$

Le dénominateur de la fonction de transfert à vide en boucle fermée admet un pôle quasiment à l'origine ( $\mathrm{si} d_{1}<10$ ) et deux pôles que nous pourrons fixer par les valeurs des coefficients $d_{0}$ et $d_{1}$. Les pôles en $p$ que nous nous fixons sont donnés par :

$$
\begin{gathered}
p_{1}, p_{1}^{*}=-\zeta \cdot \omega_{n} \pm j \omega_{p}=-2470 \pm j \cdot 2520 \\
\omega_{p}=\sqrt{1-\zeta^{2}} \cdot \omega_{n}=2513 \mathrm{rd} / \mathrm{s} \quad \text { et } \quad \zeta=0,7 .
\end{gathered}
$$

Compte tenu de la période d'échantillonnage, les pôles en $z$ correspondants seront :

$$
z_{1}, z_{1}^{*}=0,326 \pm j \cdot 0,328 \text { avec } T_{\mathrm{e}}=312,5 \mu \mathrm{s} .
$$

Il convient donc d'identifier les deux expressions suivantes :

$$
z\left(z-z_{1}\right)\left(z-z_{1}^{*}\right)=z\left(z^{2}-0,65 z+0,21\right)
$$

et

$$
z\left[z^{2}+z\left(0,186 \cdot d_{0}-1,728\right)+0,186 \cdot d_{1}+0,0001 \cdot d_{0}+0,915\right] \text {. }
$$

Après calculs, nous obtenons :

$$
d_{0}=5,8 \quad d_{1}=-3,78 .
$$

La précision de l'asservissement est étudié par le théorème de la valeur finale à l'expression (10),

$$
\lim _{z \rightarrow 1} H_{\mathrm{BF}}(z) \frac{z}{z-1}=1
$$

il vient alors :

$$
1=\frac{0,0952 \cdot\left(d_{0}+d_{2}\right)+\left\lfloor\left(d_{0}+d_{2}\right) \cdot 0.09235+0,0952 \cdot d_{1}\right\rfloor+0,09235 \cdot d_{1}}{\left[1+\left(0,1874 \cdot d_{0}-1,727\right)+\left(0,1864 \cdot d_{1}+0,915+0,0001 \cdot d_{0}\right)-5,9 \times 10^{-4} d_{1}\right]}
$$


et avec les valeurs de $d_{0}$ et $d_{1}$ calculées précédemment : $d_{2} \cong 1$. Cette valeur particulière présente l'avantage d'être pratiquement indépendante des coefficients de correction PD. Nous aboutissons ainsi à un découplage des aspects précision et stabilité, qui sont alors réglés indépendamment. Un calcul identique effectué sur charge minimale de $3 \Omega$ nous donne $d_{2} \cong 1,17$. L'atténuation étant la plus forte en charge, c'est donc à partir de sa valeur minimale $3 \Omega$ (pire des cas) que nous calculerons $d_{2}=1,17$. Nous avions évoqué précédemment l'influence de la transmittance $D_{2}(z)$ sur les zéros de la boucle fermée. La figure 8 retrace l'évolution de l'amplitude du premier dépassement sur la réponse indicielle à vide (pire des cas) en fonction de la valeur $d_{2}$. Elle montre que celui-ci décroît à mesure que la constante $d_{2}$ augmente d'où notre choix pour $d_{2}$, valeur aussi élevée que possible.

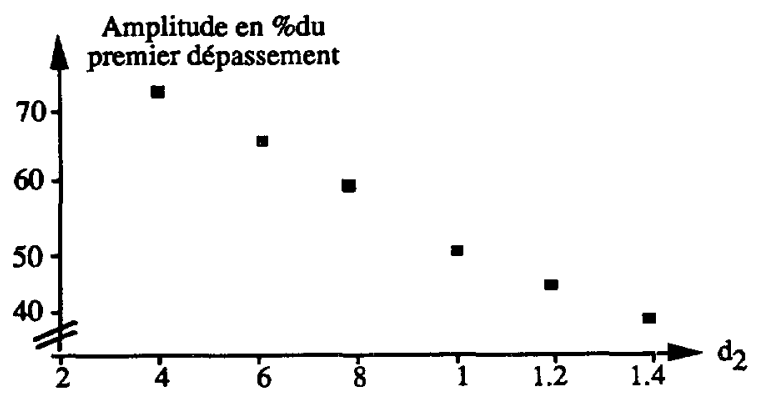

Fig. 8. - Amplitude du $1^{\text {er }}$ dépassement en fonction du facteur d'anticipation $d_{2}$.

[Overshoot versus feedforward factor $d_{2}$.]

Nous retiendrons ici la valeur particulière $d_{2}=1,17$ qui présente ainsi l'avantage de satisfaire un compromis de stabilité optimale et de précision. Mais une constatation s'imposera à nous : la compensation ne sera qu'imparfaite ! Toute brutale variation de l'angle de charge ne peut évidemment être compensée qu'après avoir été détectée, la saturation des largeurs d'impulsions qui intervient rapidement lors de l'appel de courant, limite l'efficacité de notre compensation. Il devient donc indispensable d'adjoindre une correction supplémentaire qui tiendra compte, du «passé » du système.

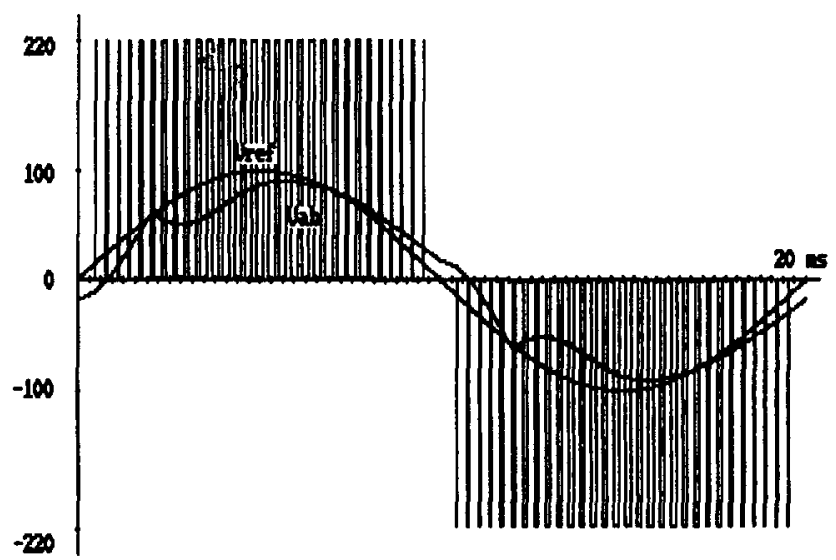

Fig. 9. - Tension de sortie en boucle ouverte. $3 \Omega$ sur $\left[36^{\circ}-180^{\circ}\right]$ et $\left[216^{\circ}-360^{\circ}\right]$, $V_{1}=88,5 \mathrm{~V}, \tau=11,5 \%$.

[Open loop waveform. $3 \Omega$ over $\left[36^{\circ}-180^{\circ}\right]$ and $\left[216^{\circ}-360^{\circ}\right], V_{1}=88.5 \mathrm{~V}, \tau=11.5 \%$.] 


\section{Pré-compensation des effets des non-linéarités.}

4.1 PRINCIPE DE PRÉ-COMPENSATION. - En admettant une périodicité de la non-linéarité (charge résistive $R_{\text {load }}$ Fig. 1), il est possible d'anticiper sur le comportement du système. Nous allons lui fournir un surplus d'énergie qui sera stocké dans le filtre LC avant l'apparition de la discontinuité et utilisé lorsque celle-ci surviendra. Pour mieux compenser les variations de la charge qui se répètent à chaque période, la régulation va les anticiper par une sorte d'autoapprentissage.

A chaque instant d'échantillonnage $k T_{\mathrm{e}}$ dans la période numéro $n$, un terme supplémentaire est ajouté dans l'algorithme de contrôle. Il permet d'augmenter artificiellement l'erreur à l'instant $k T_{\mathrm{e}}$, pour forcer la tension de sortie à dépasser sa référence. Dans l'intervalle suivant $(k+1) T_{\mathrm{e}}$, lorsque la discontinuité périodique survient « la tension de sortie chutera moins par rapport à sa référence, puisqu'elle sera partie de plus haut ». Le terme supplémentaire est fonction de l'erreur suivante lors de l'instant d'échantillonnage suivant $(k+1) T_{\mathrm{e}}$ survenue lors de la période précédente, c'est-à-dire durant la période numéro $(n-1) T_{\mathrm{e}}$, soit $(N-1)$ échantillons avant l'instant $k T_{\mathrm{e}}$. Ce terme de pré-compensation $\varepsilon_{(k-N+1)}$ illustré par la figure 10 , avec $N$ intervalles par période du signal de référence.

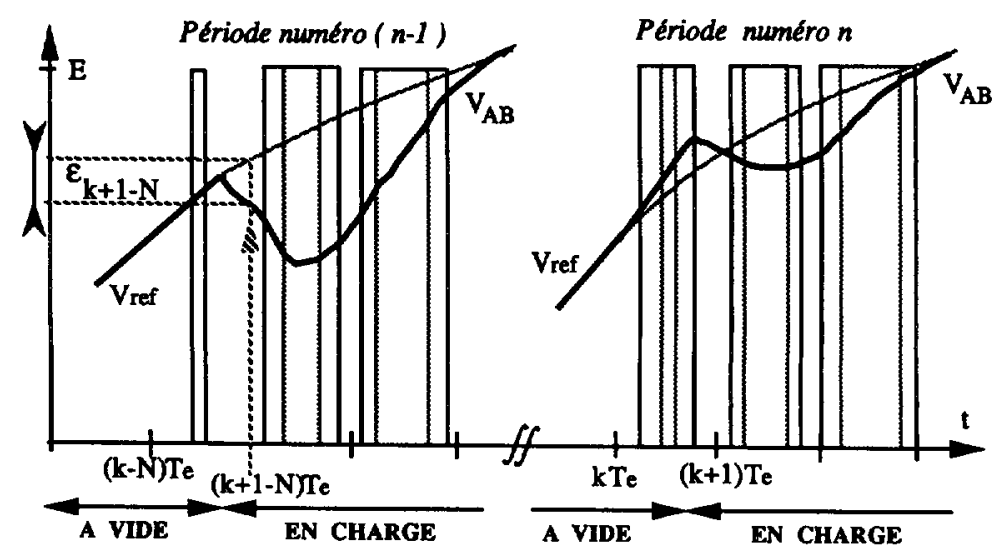

Fig. 10. - Principe de pré-compensation des variations périodiques de la charge.

[Principle of the pre-compensation of cyclic fluctuatings loads.]

La modélisation de cette action de précompensation par la transformée en $z$ peut-être écrite simplement en considérant que le terme correctif n'est qu'une valeur de l'erreur, mémorisée $N-1$ échantillons auparavant. Il vient donc alors (10) :

$$
\varepsilon^{\prime}(z)=\varepsilon(z)+d_{3} \cdot z^{-(N-1)} \quad \varepsilon(z) \quad \text { d'où } \quad D_{3}(z)=\frac{\varepsilon^{\prime}(z)}{\varepsilon(z)}=\left(1+d_{3} \cdot z^{-N+1}\right) .
$$

C'est sur ce terme d'erreur modifié $\varepsilon^{\prime}(z)$ que s'applique la correction PD énoncée dans la formule (7). Il est possible de représenter cette action d'anticipation sous la forme d'une correction cascade supplémentaire $D_{3}(z)$ dans le schéma-blocs figure 11 .

Il est alors possible d'écrire l'expression de la grandeur de commande $U(z)$ en fonction des valeurs de la référence et de l'erreur modifiée $\varepsilon^{\prime}(z)$ :

$$
U(z)=d_{2} \cdot V_{\mathrm{ref}}(z)+d_{0} \cdot \varepsilon^{\prime}(z)+d_{1} \cdot \varepsilon^{\prime}(z) \cdot z^{-1}
$$




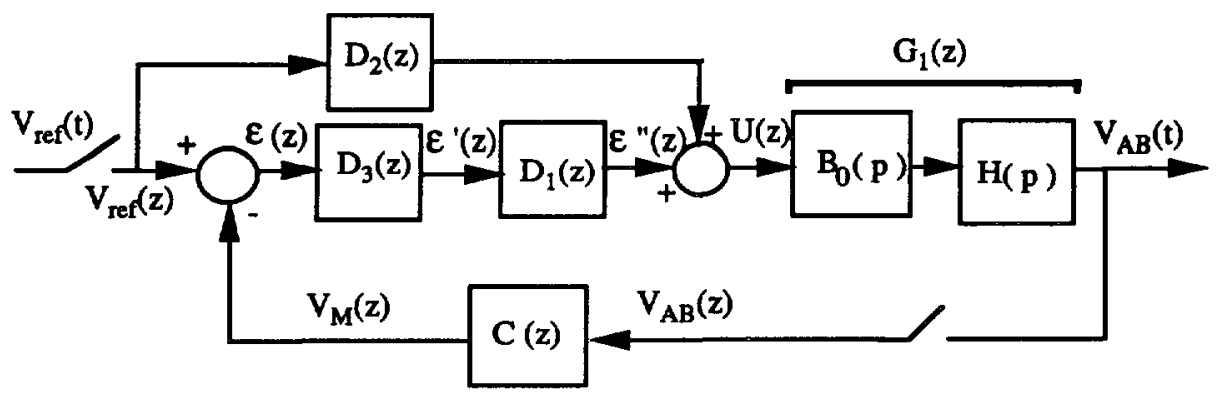

Fig. 11. - Schéma-blocs de l'action de pré-compensation.

[Blocs diagram of pre-compensation action.]

soit

$$
U(z)=d_{2} \cdot V_{\mathrm{ref}}(z)+D_{1}(z) \cdot \varepsilon^{\prime}(z) .
$$

Pour obtenir un comportement reproductible d'une période sur l'autre, nous sommes amenés à ajouter dans l'algorithme deux termes supplémentaires $\varepsilon_{k-N}$ et $\varepsilon_{k-2 N}$. Ils font intervenir pour le calcul de la largeur d'impulsion à l'instant $k$, les erreurs mémorisées durant le même intervalle lors des deux périodes précédentes, numérotées $(n-1)$ et $(n-2)$.

Il s'agit d'une sorte de sommation avec oubli progressif du passé, puisque l'on ne retient que l'erreur instantanée et les valeurs sur les deux périodes passées. La figure 12 repère la position des différents échantillons qui serviront de base au calcul de la largeur d'impulsion à l'instant $k T_{\mathrm{e}}$. Le principe de cette pré-compensation supplémentaire s'apparente à une action de type intégral. En effet, c'est pour chaque intervalle $k$ qu'a lieu cette « intégration », par sommation des valeurs de l'erreur dans les intervalles $k$ de chaque période précédente. Le facteur d'oubli permettra de donner une plus grande importance aux deux dernières périodes écoulées. Nous limitons ici volontairement l'ordre de la sommation du terme « intégral » à 2 , ce qui permet d'obtenir une précision suffisante en régime permanent tout en privilégiant la rapidité de réaction lors d'une variation de la charge.

Cette sommation peut se modéliser en ajoutant deux termes à l'expression $\varepsilon^{\prime}(z)$ définie dans l'expression (11), pour obtenir le correcteur définitif $D_{3}(z)$, notée dans (13) :

$$
\varepsilon^{\prime}(z)=\varepsilon(z)+d_{3} \cdot z^{-(N-1)} \quad \varepsilon(z)+d_{4} \cdot z^{-N} \quad \varepsilon(z)+d_{5} \cdot z^{-2 N} \quad \varepsilon(z)
$$

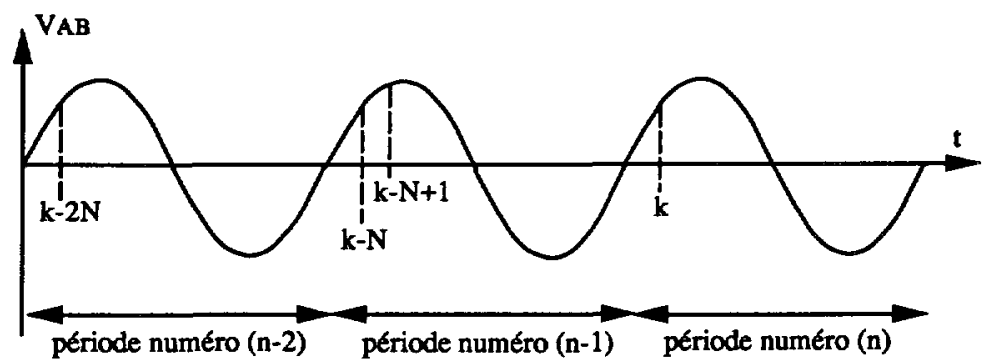

Fig. 12. - Echantillons utilisés pour la pré-compensation complète.

[Samples used for the complete pre-compensation.] 
d'où

$$
D_{3}(z)=\frac{\varepsilon^{\prime}(z)}{\varepsilon(z)}=\left(1+d_{3} \cdot z^{-N+1}+d_{4} \cdot z^{-N}+d_{5} \cdot z^{-2 N}\right)
$$

A partir du schéma blocs de la figure 11 et de la formule (13), nous pouvons maintenant définir parfaitement l'expression de la grandeur de commande $U(z)$ :

$$
U(z)=d_{2} \cdot V_{\text {ref }}(z)+D_{1}(z) \cdot D_{3}(z) \cdot \varepsilon(z)
$$

soit en reportant (6) et (11) dans (14), il vient finalement (15), expression assez simple qui sera implantée dans le calculateur pour réaliser l'algorithme de commande de l'onduleur :

$$
U(z)=d_{2} \cdot V_{\text {ref }}(z)+\left(d_{0}+d_{1} \cdot z^{-1}\right) \cdot\left(1+d_{3} \cdot z^{-(N-1)}+d_{4} \cdot z^{-N}+d_{5} \cdot z^{-2 N}\right) \cdot \varepsilon(z) .
$$

4.2 CAlCul DE L'ACTION DE PRÉ-COMPENSATION. - Le critère de calcul retenu pour cette action de prédiction est illustré par la figure 13. La valeur moyenne de la tension $V_{\mathrm{AB}}$ avec pré-compensation devra être égale à la valeur de référence. Il convient donc de modifier localement la pente du signal de référence, c'est-à-dire la largeur de l'impulsion correspondante.

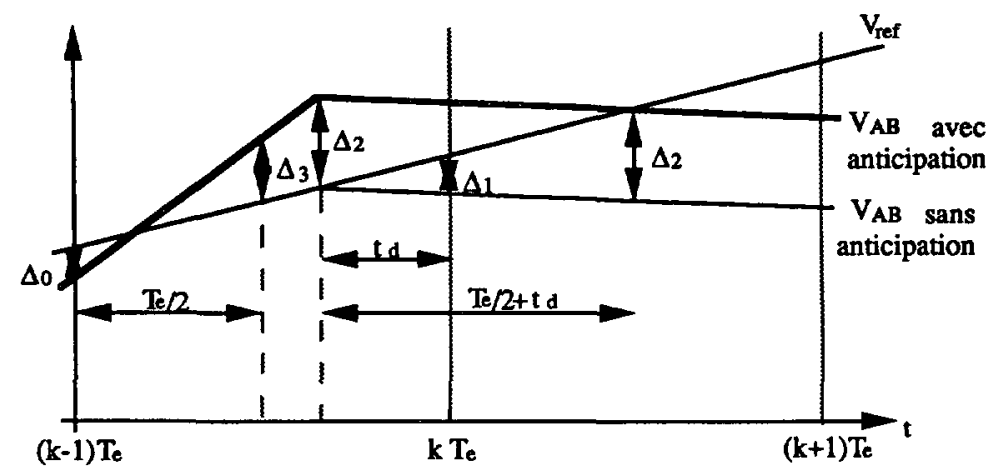

Fig. 13. - Principe de calcul du coefficient de pré-compensation.

[Method to determine the coefficient of pre-compensation.]

Le calcul du coefficient de pré-compensation découle d'un critère géométrique. Si l'on désire qu'avec l'action d'anticipation la grandeur de sortie soit confondue avec la référence au milieu de l'intervalle, il faut ajouter à l'instant $(k-1) T_{\mathrm{e}}$ un supplément de commande $\Delta_{3}$, fonction de l'erreur non compensée $\Delta_{1}$ détectée lors de la période précédente $(n-1)$ à l'instant $k T_{\mathrm{e}}$. En approximant les courbes par des droites sur chaque intervalle, cette modification revient à ajouter une largeur d'impulsion correspondant à $\Delta_{3}$ sur l'impulsion précédant l'intervalle où fut mesurée l'erreur $\Delta_{1}$, lors de la période précédente.

$$
\Delta_{3}=\frac{T_{\mathrm{e}} \cdot\left(2 \cdot \frac{t_{\mathrm{d}}}{T_{\mathrm{e}}}+1\right)}{4 \cdot t_{\mathrm{d}} \cdot\left(\frac{t_{\mathrm{d}}}{T_{\mathrm{e}}}-1\right)} \cdot \Delta_{1}
$$

il vient alors $d_{3}=0,4$. 
L'algorithme ainsi modifié réalise une pré-compensation par prédiction. Le comportement du système est donc mémorisé lors d'une période, puis corrigé lors de la suivante. Ceci suppose évidemment que les caractéristiques de la charge n'ont pas varié d'une période sur l'autre. Cette mémorisation de l'erreur lors des deux périodes précédentes, constitue donc un " auto-apprentissage progressif " de la charge. Les valeurs des coefficients $d_{4}$ et $d_{5}$ sont déterminées expérimentalement à partir de plusieurs essais sur des charges non linéaires différentes avec $d_{3}=0,4$, on obtient : $d_{4}=0,3 ; d_{5}=0.15$.

4.3 RÉSUltatS DE SIMULATION AVEC ACTION DE PRÉ-COMPENSATION. - Les essais présentés avec différentes charges non linéaires, valident notre étude. Les figures $14 \mathrm{a}$ et $14 \mathrm{~b}$ donnent les résultats pour une même valeur de la charge, mais avec des angles de commutation différents. Les coefficients de l'algorithme de compensation sont: $d_{0}=5,8 ; d_{1}=-3,8 ; d_{2}=1,17$; $d_{3}=0,4 ; d_{4}=0,3 ; d_{5}=0,15$. La robustesse du correcteur vis-à-vis de la charge est confirmée puisque les coefficients sont les mêmes dans les deux cas.

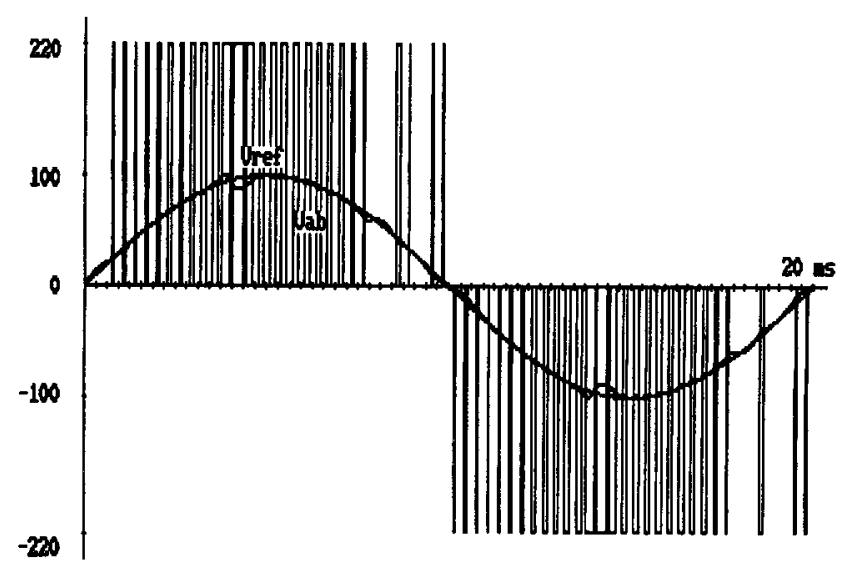

a)

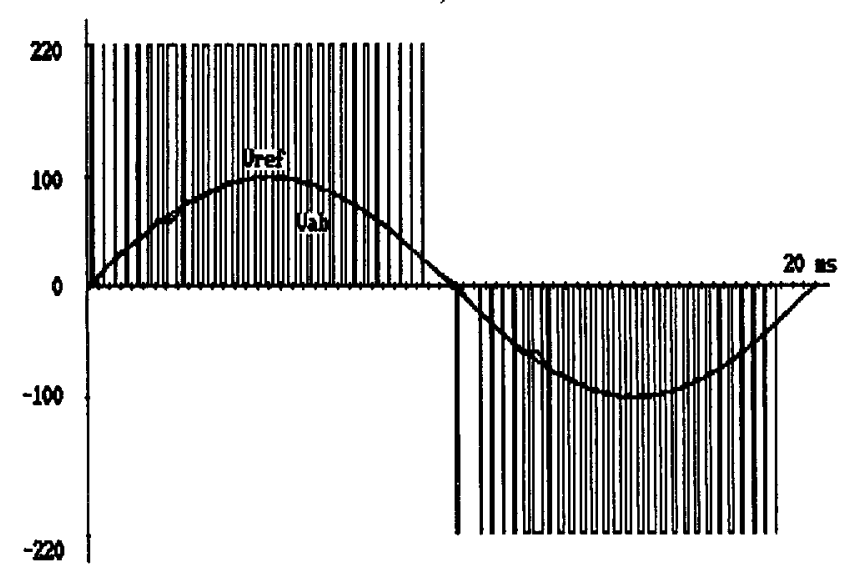

b)

Fig. 14. - Comparaison des formes d'ondes pour deux charges non linéaires. a) $3 \Omega$ sur $\left[70^{\circ}-140^{\circ}\right]$ et $\left[250^{\circ}-320^{\circ}\right] V_{1}=99,4 \mathrm{~V}, \tau=4,3 \%$. b) $3 \Omega$ sur $\left[36^{\circ}-180^{\circ}\right]$ et $\left[216^{\circ}-360^{\circ}\right], V_{1}=99,3 \mathrm{~V}, \tau=2,6 \%$.

[Comparison of closedloop waveforms under two non linear loads (a, b). a) $3 \Omega$ over $\left[70^{\circ}-140^{\circ}\right.$ ] and $\left[250^{\circ}-320^{\circ}\right] V_{1}=99.4 \mathrm{~V}, \tau=4,3 \%$. b) $3 \Omega$ over $\left[36^{\circ}-180^{\circ}\right]$ and $\left[216^{\circ}-360^{\circ}\right] V_{1}=99.3 \mathrm{~V}, \tau=2.6 \%$. $]$ 
Les résultats obtenus sur ces charges non linéaires valident le principe de notre commande. Néanmoins, la limite de cette méthode est ici atteinte puisque le phénomène de saturation des largeurs d'impulsion persiste, bien que fortement minimisé. Il constitue un inconvénient technologique qu'il sera fort difficile de surmonter. Une étude en simulation a montré [13], qu'il existe plutôt un domaine de valeurs optimales, où la valeur de $t_{\mathrm{c}}$ n'influence que peu les résultats sur le taux de distorsion de l'onde de sortie.

Dans la zone autour d'une trentaine de microsecondes, le taux de distorsion est minimum. C'est donc la valeur que nous retiendrons dans cette étude. Le temps $t_{\mathrm{c}}$ étant faible devant la période d'échantillonnage $t_{\mathrm{c}} \ll T_{\mathrm{e}}$, nous pouvons valider le principe de mesure comme un véritable capteur de pente à chaque instant d'échantillonnage.

4.4 PERFORMANCES EN ROBUSTESSE. - Le caractère extrêmement contraignant des charges non linéaires employées ici est à l'origine de la saturation observée. Si la résistance de charge est plus grande, par exemple $5 \Omega$, elle entraîne une perturbation moindre sur la tension de sortie de l'onduleur et les résultats sont bien meilleurs comme en témoignent les figures $15 \mathrm{a}$ et $b$.

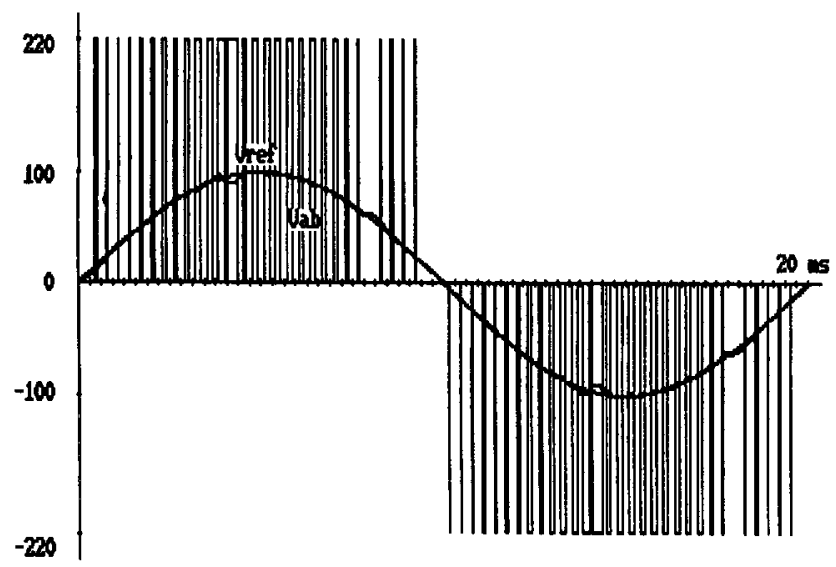

a)

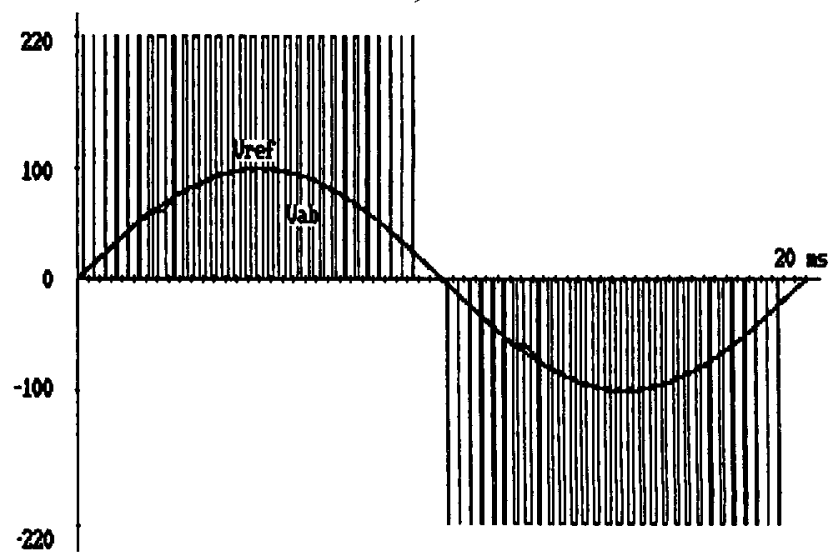

b)

Fig. 15. - Forme d'onde en boucle fermée pour une charge non linéaire de $5 \Omega$. a) $5 \Omega$ sur $\left[70^{\circ}-140^{\circ}\right]$ et $\left[250^{\circ}-320^{\circ}\right], V_{1}=99 \mathrm{~V}, \tau=3 \%$. b) $5 \Omega$ sur $\left[36^{\circ}-180^{\circ}\right]$ et $\left[216^{\circ}-360^{\circ}\right], V_{1}=100,6 \mathrm{~V}, \tau=2,4 \%$.

[Closed loop wave forms, under a non linear load of $5 \Omega$. a) $5 \Omega$ over $\left[70^{\circ}-140^{\circ}\right]$ and $\left[250^{\circ}-320^{\circ}\right]$, $V_{1}=99 \mathrm{~V}, \tau=3 \%$. b) $5 \Omega$ over $\left[36^{\circ}-180^{\circ}\right]$ and $\left[216^{\circ}-360^{\circ}\right], V_{1}=100.6 \mathrm{~V}, \tau=2.4 \%$.] 
Pour cette valeur de résistance de charge l'amortissement est plus important donc le système est plus stable. L'appel de courant lors de la connexion de la résistance de charge est plus faible, la tension de sortie chute donc naturellement moins vers $70^{\circ}$.

La compensation a été calculée pour le pire des cas, c'est-à-dire pour le fonctionnement à vide donc à amortissement minimal. Ces déterminations ont été faites sans préjuger des angles de commutation de la charge non linéaire. Les figures $16 \mathrm{a}$ et $16 \mathrm{~b}$ présentent les formes d'onde obtenues lors d'un régime transitoire de la charge, lors d'une variation brutale des angles de commutation qui passent d'une valeur initiale de $\left[70^{\circ}-140^{\circ}\right]-\left[250^{\circ}-320^{\circ}\right]$ lors de la période $n$, à $\left[36^{\circ}-180^{\circ}\right]-\left[216^{\circ}-360^{\circ}\right]$ lors des périodes suivantes.

Le retour au régime permanent convenablement compensé, l'apprentissage de la nouvelle configuration de la charge et l'oubli de l'ancienne sont terminés en 3 périodes du signal de référence, c'est-à-dire en $60 \mathrm{~ms}$. Les figures 16 soulignent donc les performances de la

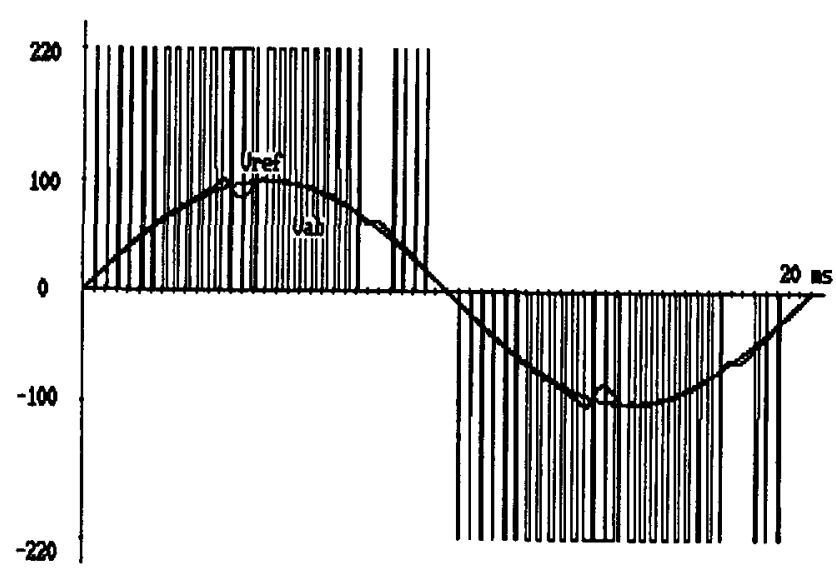

a)

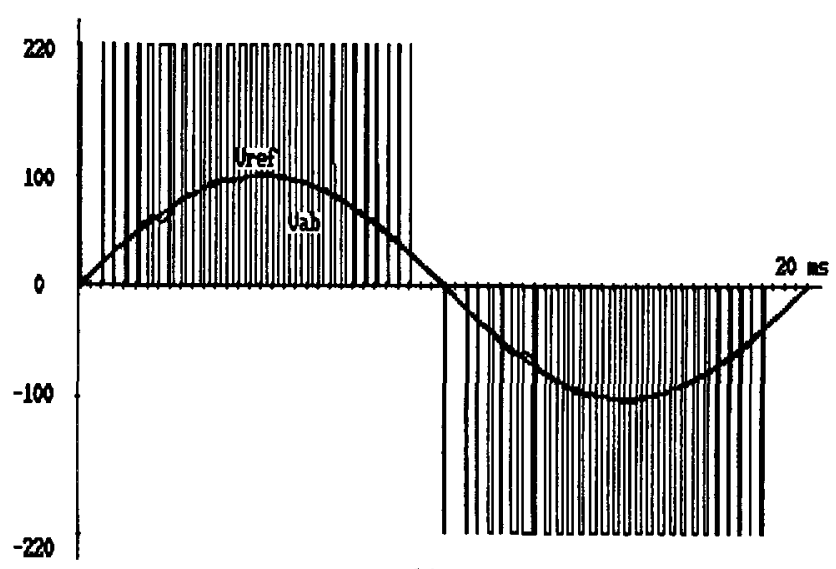

b)

Fig. 16. - Evolution de la tension de sortie de l'onduleur lors d'une brutale variation des caractéristiques de la charge non linéaire. a) Période $n, 3 \Omega$ sur $\left[70^{\circ}-140^{\circ}\right]$ et $\left[250^{\circ}-360^{\circ}\right], V_{1}=100,9 \mathrm{~V}, \tau=3.52 \%$. b) Période $n+3,3 \Omega$ sur $\left[36^{\circ}-180^{\circ}\right]$ et $\left[216^{\circ}-360^{\circ}\right], V_{1}=101,2 \mathrm{~V}, \tau=2,6 \%$.

[Output voltage in the presence of an extreme variation of the nonlinear load. a) Period $n, 3 \Omega$ over $\left[70^{\circ}\right.$ $\left.140^{\circ}\right]$ and $\left[250^{\circ}-360^{\circ}\right], V_{1}=100.9 \mathrm{~V}, \tau=3.52 \%$. b) Period $n+3,3 \Omega$ over $\left[36^{\circ}-180^{\circ}\right]$ and $\left[216^{\circ}-360^{\circ}\right]$, $V_{1}=101.2 \mathrm{~V}, \tau=2.6 \%$.] 
commande lors d'un « régime dynamique de la charge », tandis que les figures 14a et $14 \mathrm{~b}$ présentaient la compensation de différentes valeurs de charge en régime permanent. De plus, la valeur du fondamental de l'onde sinusoïdale est régulée autour d'une valeur de référence de $100 \mathrm{~V}$, sans boucle nécessité d'une boucle de régulation spécifique, solution proposée dans d'autres publications [18]. L'ensemble de ces résultats confirment l'efficacité et la rapidité de la compensation des effets des charges non linéaires par notre commande temps réel d'onduleur MLI.

\section{Conclusion.}

La nouvelle méthode de régulation présentée dans cet article, pour le contrôle instantané d'un onduleur MLI, possède beaucoup d'avantages et permet d'obtenir de très bonnes performances sur charges non linéaires. La régulation est capable de compenser aussi bien les harmoniques pairs que les harmoniques impairs, donc tout type de charge non linéaire.

Grâce à un algorithme simple, le temps de calcul est réduit au minimum et le retard de réaction après chaque échantillonnage est très faible, sa simplicité (quelques additions et multiplications) permet d'envisager aisément son implantation sur tout microprocesseur 16 bits. Une réalisation pratique sera développée au laboratoire à base de microprocesseurs traitement du signal.

La correction par prédiction avec une mémorisation limitée à l'ordre 2, permet d'obtenir à la fois une bonne précision en régime permanent sinusoïdal et une réaction rapide en régime dynamique, c'est-à-dire en cas de changement en cours de fonctionnement des caractéristiques de la charge non linéaire. Cette méthode utilise une modélisation unique de la charge, du filtre et de l'onduleur par la transformation en $z$. Elle ne nécessite qu'un seul calcul préalable des coefficients du modèle et des correcteurs pour le fonctionnement à vide, cas le plus défavorable.

Pour protéger l'onduleur contre un court-circuit, un temps de déconnexion est obligatoire entre la commande des deux interrupteurs de chaque bras. Ces temps morts créent une distorsion harmonique non négligeable et modifient la valeur du fondamental de la tension de sortie. Ils sont considérés par la régulation comme une non-linéarité supplémentaire et sont compensés de la même façon.

De part les performances actuelles de interrupteurs statiques de puissance, la fréquence de commutation employée dans cette étude convient aussi bien aux transistors bipolaires qu'aux transistors MOS. La grande simplicité et la robustesse de cette méthode de régulation en font un principe particulièrement bien adapté aux alimentations sans coupures, pour de nombreuses applications aéronautiques et informatiques.

\section{Bibliographie}

[1] IEEE Working Group on Power System Harmonics « Power System Harmonics : an Overview 》, IEEE Trans. Pow. Appar. Syst. PAS-102 (1983) 2455-2460.

[2] Linders J. R., Electric Wave Distorsions: Their Hidden Costs and Containment, IEEE Trans. Ind. Appl. IA-15 (1979) 458-471.

[3] Patel H. S. and Hoft R. G., Generalized Technique of Harmonic Elimination and Voltage Control in Thyristor Inverter-Part I, IEEE Trans. Ind. Appl. IA-9 (1973) 310-317 and Part II, IA-10 (1973) 666-673.

[4] Sournac E., Hapiot J. C., Maussion P., Sabourrin C., Implementation of Optimised Modulation in an Industrial Speed Drive for Asynchronous Machine EPE, Aachen (1989). 
[5] Richards G. G., Klinkhachorn P., Tan O. T. and Hartana R. K., Optimal LC Compensators for Nonlinear Loads with Uncertain Nonsinusoïdal Source and Load Characteristics, IEEE Trans. Power Syst. 4 (1989) 30-36.

[6] Chauveau B., Etude de la commande d'un onduleur alimentant une charge non linéaire à partir d'une source impédante : optimisation de la distorsion harmonique de sa tension de sortie, Thèse de Docteur Ingénieur-LEEI-ENSEEIHT-Institut National Polytechnique de Toulouse (1987).

[7] Kawaramura A. and Hoft R., Instantaneous Feedback Controlled PWM Inverter with Adaptative Hysteresis, IEEE Trans. Ind. Appl. IA-20 (1984) 769-775.

[8] Haneyoshi T., Kawamura A. and Hoft R., «Waveform Compensation of PWM Inverter with Cyclic Fluctuating Loads», IEEE Ind. App. Annual Meeting (1986) pp. 744-751.

[9] Chung Y. H., Kim S. S., Cha H. J., Kang M. G. and Sul S. K., Parallel Operation of Voltage Inverters by Real-Time Digital PWM Control, IEEE Ind. App. Annual Meeting, EPE Congress, Firenze, Italy (1991) pp. 1058-1063.

[10] Faucher J. and Grandpierre M., « New Methodological Concepts for Systems Simulation : Application to a Controlled Converter Machine », 12th IMACS Worls Congress-Paris-France (July 1988).

[11] Bordry F. and Foch H., « Simulation of semiconductor electrical networks », 11 th INMACS World Congress-Oslo-Norway (August 1985).

[12] Widodo T., Etude de convertisseurs statiques de fréquence, Thèse de Docteur Ingénieur-LEEIENSEEIHT-Institut National Polytechnique de Toulouse (1981).

[13] Maussion P., Commande numérique d'un onduleur de tension en modulation de largeur d'impulsion sur charges non linéaires. Thèse de Docteur de l'Institut National Polytechnique de ToulouseLEEI (1989).

[14] Gille J. C., Decaulne P., Pellegrin M., Théorie et calcul des asservissements (Dunod).

[15] Kuo B. C., Automatic Control Systems (Prentice Hall).

[16] Astrom K. J., Witemark B., Computer Controlled Systems (Prentice-Hall, 1990).

[17] Bühler H. R., Réglages échantillonnés (Presses Polytechniques Romandes, Lausanne).

[18] Kawamura A., Yokoyama T., « Comparaison of Five Control Methods for Digitally Feedback Control PWM Inverters », EPE Congress, Firenze-Italy (1991) pp. 2035-2040. 